\title{
Perfíl lipídico en una comunidad de Calarcá, Colombia
}

\author{
Andrea Arias', Mery Castillo, Patricia Landázuri², Beatriz Restrepo², Martha L. Gallego²
}

\begin{abstract}
Resumen
Las enfermedades cardiovasculares (EC), en general, y la cardiopatía isquémica, en particular, constituyen un importante problema de salud pública por su alta mortalidad, morbilidad, costos médico-hospitalarios e impacto laboral por su poder incapacitante. Estas patologías constituyen la primera causa de muerte en la población adulta en Colombia después de las muertes por violencia. Dado que, en nuestro país, no se han determinado con exactitud parámetros propios del comportamiento de los lípidos sanguíneos en la población, se hace necesaria su caracterización para que se puedan establecer políticas de intervención para la prevención y control de EC, tanto a nivel individual como poblacional. Este trabajo intenta contribuir al estudio poblacional del comportamiento de los niveles de lípidos en poblaciones colombianas. Se determinaron los niveles de colesterol total (CT), colesterol en lipoproteínas de baja densidad (C-LDL), colesterol en lipoproteínas alta densidad (C-HDL) y triglicéridos (TG), en sueros de 71 varones escogidos al azar con edades comprendidas entre 20 y 70 años. Se utilizaron métodos enzimáticos con reactivos Boehringer Manheinn. Los resultados de este estudio muestran que 21,1\% de la población presenta colesterol mayor de $200 \mathrm{mg} / \mathrm{dL}, 66,2 \%$ C-HDL menor de $35 \mathrm{mg} / \mathrm{dL}, 5,6 \%$ presentan C-LDL mayor de $150 \mathrm{mg} / \mathrm{dL}$ y 36,6 presenta TG mayor de $150 \mathrm{mg} / \mathrm{dL}$. Estos datos indican que, para esta población, el factor protector para las EC (C-HDL) está seriamente afectado y, además, presenta una hipertrigliceridemia aislada. Se encontró una alta incidencia sobre los valores del perfil lipídico de los factores de riesgo no lipídicos como son el índice de masa corporal, el fumar, los antecedentes familiares y la edad. Los resultados obtenidos en el presente estudio se semejan a los encontrados para poblaciones de países en desarrollo y los encontrados en estudios de poblaciones colombianas, por tal motivo deben iniciarse estrategias de prevención y educación para el control de la EC.
\end{abstract}

\section{Summary}

Heart disease (HD) in general and ischemic cardiopathy in particular constitute an important public health problem due to the impact of their high mortality, morbidity, medical costs and lost workdays due to its incapacitating influence. These pathologies constitute the major cause of death in the Colombian adult population, following violent deaths.

Several regional studies of plasma lipid behaviour in the population have been made, however their characterisation in other regions in order to establish supervisory policies for HD prevention and control at individual as well as whole population level has been made necessary. This work attempts to contribute to lipid level behaviour study in Colombian populations.

Programa de biología y educación ambiental. Universidad del Quindío.

2. Centro de investigaciones biomédicas "Manuel Elkin Patarroyo". Facultad de Medicina, Universidad del Quindío. 
Total cholesterol levels (TC), cholesterol in high density lipoproteins (C-HDL), cholesterol in low density lipoproteins (C-LDL) and triglycerides (TG) were determined in 71 male volunteers' serum, ages ranging from 20 to 70 years old. Enzymatic methods were employed (Boehringer Manheinn reagents). This study's results show that $21.1 \%$ of the population present TC higher than $200 \mathrm{mg} / \mathrm{dL}, 66.6 \% \mathrm{C}$-HDL under $35 \mathrm{mg} / \mathrm{dl}, 5.6 \% \mathrm{C}$-LDL higher than $150 \mathrm{mg} / \mathrm{dL}$ and $36.6 \%$ presented TG higher than $150 \mathrm{mg} / \mathrm{dL}$. This data shows that for this population the $\mathrm{CHD}(\mathrm{C}-\mathrm{HDL})$ protective factor is seriously affected and furthermore presents an isolated hypertriglyceridemia. High incidences of non-lipid risk factors, such as bodymass, smoking, family antecedents and age, were recorded on the lipid profile's values. The results obtained in this study are like those found in developing countries' populations and are also like those found in Colombian population studies. Therefore, preventative and educational strategies must be implemented to control HD.

La enfermedad cardiovascular (EC) constituye un problema de salud pública grave por su alta morbimortalidad, su poder incapacitante y sus altos costos por la demanda de atención e incapacidades que genera, todo lo cual tiene una . gran repercusión socioeconómica y familiar.

El estudio de Framminghan (1) así como otros estudios epidemiológicos $(2,3)$ han mostrado que existe una asociación entre las EC y determinados factores de riesgo, lipídicos y no lipídicos, los cuales a su vez están condicionados por el estilo de vida y el ambiente del individuo y de las sociedades.

En relación con los factores lipídicos de riesgo, numerosos estudios epidemiológicos $(2,4)$ demostraron que la morbimortalidad cardiovascular aumenta a medida que aumenta el valor del colesterol total (CT), el colesterol transportado en las lipoproteínas de baja densidad (C-LDL) o los triacilglicéridos (TG) y a medida que disminuye el valor del colesterol transportado en las lipoproteínas de alta densidad (C-HDL). Está plenamente comprobado que el colesterol y, en particular, el contenido en las LDL interviene directamente en la formación de la placa ateromatosa; por el contrario, las HDL se identifican como un factor protector para la reducción de la aterogénesis. La relación CT/ HDL se maneja hoy como un buen indicador de riesgo ya que refleja la relación entre las dos subfracciones; esta relación ha resultado ser el mejor dato pronóstico para las EC (5).

Los TG en la mayoría de los estudios epidemiológicos constituyen un factor de riesgo independiente, pero siempre deben analizarse en conjunto con los niveles de HDL; actualmente, se sabe que la hipertrigliceridemia induce a cambios profundos en la composición de las lipoproteínas, incluyendo HDL y LDL. Hay una correlación entre la hipertrigliceridemia y niveles reducidos de HDL2 y entre hipertrigliceridemia y altos niveles de $\operatorname{LDL}(6,7)$.

En algunos países de Latinoamérica (8) y en Colombia (9), las EC constituyen la primera causa de muerte no violenta. Entre 1981 y 1984, países como Argentina y República Dominicana (8) alcanzaron cifras de muerte por EC mayores que Estados Unidos; en Colombia, las muertes por EC pasaron de $5 \%$, en 1970 , a $27 \%$ en 1991 , según el Ministerio de Salud, lo cual indica que las EC dejaron de ser solo un problema de los países industrializados y pasó a serlo también de los países en desarrollo los cuales están experimentando profundos cambios en su estilo de vida.

En Colombia, existen algunos estudios publicados que revelan la prevalencia de EC, el más importante de ellos realizado por el Instituto Nacional de Salud (INS) entre 1978 y 1982 reveló que $10,4 \%$ de los hombres y $16 \%$ de las mujeres mayores de 15 años tenían colesterol superior a 240 $\mathrm{mg} / \mathrm{dL}$. En un estudio de 1992, realizado en la ciudad de Pereira (10) y que implicó el perfil lipídico completo, se registraron cifras muy parecidas a las informadas por el INS, $15 \%$ de la población estudiada presentaba niveles de colesterol superiores a $240 \mathrm{mg} / \mathrm{dL}$ y triglicéridos de 235,5 $\mathrm{mg} / \mathrm{dL}$ para la población masculina. En estudios más recientes, en la población del archipiélago de San Andrés y de San Juan de Pasto, Suárez 
y colaboradores (11-13) informaron datos muy similares a los encontrados en el estudio del INS y en el estudio de Pereira, así, la hipercolesterolemia (>220 mg/dL), estuvo entre 16 y $26 \%$, en dos grupos poblacionales diferentes de la isla y los triglicéridos entre 15 y $30 \%$ para los mismos grupos.

En Quindío, no existen datos informados que permitan determinar los riesgos de la población; los resultados de este estudio aportan alguna información al respecto, además que amplían los datos existentes para la población colombiana, abriendo posibilidades de tener nuestros propios niveles de referencia y así poder establecer políticas de intervención en salud preventiva, curativa y educacional para el individuo en particular y la población en general.

\section{Materiales y métodos}

En una población total de 309 varones mayores de 20 años, todos de raza blanca, dedicados a diversas actividades, como transportadores, comerciantes o trabajadores del campo, habitantes del barrio Veracruz, de Calarcá, Quindío, se escogieron aleatoriamente 85 , un hombre por cada casa seleccionada aleatoriamente de acuerdo con la manzana del barrio; de estos individuos, se incluyeron en el estudio 71 voluntarios, el resto no acudió al centro de salud o después de llenar la encuesta no cumplían con los criterios de inclusión; éstos eran: individuos sanos (examen médico previo), sin diabetes mellitus, ni antecedentes de enfermedad cardiovascular. Se excluyeron aquellos con enfermedades cardíacas, dislipidemias y diabetes mellitus. La población de Veracruz fue escogida por ser área de influencia de un centro de salud de la Facultad de Medicina de la Universidad del Quindío.

Se tomaron muestras de $5 \mathrm{~mL}$ de sangre venosa en $200 \mu \mathrm{l}$ de EDTA, después de un ayuno de 12 horas de duración y no haber ingerido alcohol el día previo. El plasma se obtuvo por centrifugación a $4^{\circ} \mathrm{C}$. Posteriormente, se determinó colesterol total (CT), colesterol HDL (C-HDL), colesterol LDL (C-LDL) y triacilglicéridos (TG). EI C-LDL se determinó mediante la formula C-LDL=CT - (TG/5) $\mathrm{C}-\mathrm{HDL}$, para sueros que tuvieran TG inferior a 400 $\mathrm{mg} / \mathrm{dL}$. Para todas estas determinaciones, se uti- lizaron reactivos líquidos y métodos enzi-máticos de Boehringer Manheinn.

Además, a cada sujeto se le determinó la presión arterial en posición sentado y se definió como hipertenso si tenía la presión sistólica en valores por encima de $150 \mathrm{~mm} \mathrm{Hg}$. También, se determinó el índice de masa corporal(Ml=peso/altura $\left.{ }^{2}\right)$, índice arterial (IA=CT/C-HDL) y sus hábitos dietarios y físicos se registraron en una encuesta. Los hábitos dietarios se calificaron de acuerdo con el programa nacional de educación en colesterol de los Estados Unidos (11), modificada por nosotros para nuestro medio, brevemente: los alimentos se dividieron en cinco grupos: grupo 1 , carnes (cerdo, pollo con piel, res, otros); grupo 2, productos lácteos (leche entera, yogur, queso, mantequilla); grupo 3, fritos (chorizos, chicharrón, platos fritos, vísceras, otros); grupo 4, huevos; grupo 5, meriendas o entrecomidas. El puntaje se obtuvo multiplicando el consumo semanal (3 para menos de tres veces y 7 para más de cuatro veces a la semana) por el tamaño de la porción (1 para una porción pequeña, 2 para una porción normal y 3 para una porción doble o grande); ejemplo, carne de cerdo dos veces a la semana, una porción normal $3 \times 2=6$. El puntaje total es la sumatoria de todos los puntos en cada grupo de alimentos. La clasificación de riesgo se da así: ningún riesgo 40; bajo riesgo de 41 a 70 y alto riesgo, 70 puntos.

\section{Estadística}

Se utilizó análisis de regresión para determinar la incidencia de las variables independientes (fumar, presión sistólica, dieta, antecedentes familiares, edad, índice de masa) en las variables dependientes (colesterol total, triglicéridos, C-HDL, C-LDL).

\section{Resultados}

La figura 1 muestra los resultados de la encuesta dietaria; los datos indican que un alto porcentaje de la población en estudio tiene bajo riesgo (64\%) o no presenta ningún riesgo $(32,3)$ de sufrir EC, mientras que $2,8 \%$ está en alto riesgo de sufrir EC por ingestión de alimentos ricos en grasas saturadas y colesterol.

El cuadro1 indica el porcentaje de individuos de la población total con alteraciones del perfíl lipídico. 


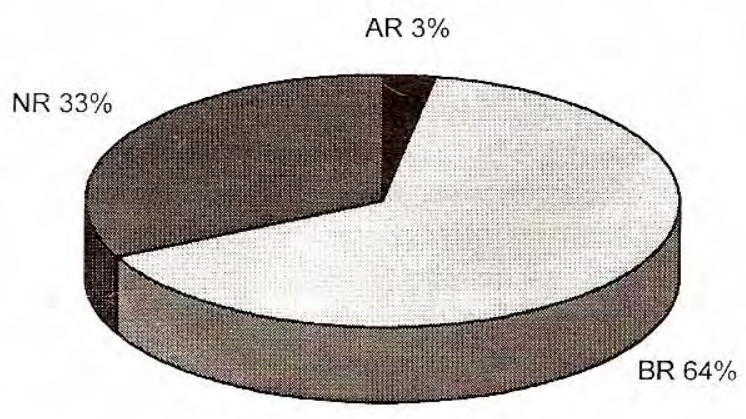

Figura 1. Nivel de riesgo de sufrir EC por ingestión de alimentos ricos en grasas saturadas.

AR: alto riesgo; BR: bajo riesgo; NR: ningún riesgo. El riesgo fue calificado de acuerdo con el programa de Educación en Colesterol de los Estados Unido, NCEP (11).

Este cuadro muestra un elevado número de individuos $(66 \%)$ con C-HDL (factor protector de riesgo) por debajo de $35 \mathrm{mg} / \mathrm{dL}$. También es notoria la hipertrigliceridemia en la población (36\%), mientras que $21 \%$ de la población tuvo colesterol por encima de $200 \mathrm{mg} / \mathrm{dL}$. Otro factor importante de riesgo que presentó la población fue el IMC

Cuadro 1. Porcentaje de individuos con alteraciones en el perfíl lipídico.

\begin{tabular}{lrc}
\hline $\begin{array}{l}\text { Perfil lipídico } \\
\text { Valores de riesgo* } \\
\text { (mg/dL) }\end{array}$ & $\begin{array}{c}\text { Individuos con alteraciones } \\
\text { del perfil lipídico }\end{array}$ \\
\hline CT $\quad \geq 200$ & 21,1 \\
C-HDL & $\leq 35$ & 66,1 \\
C-LDL & $\geq 50$ & 5,6 \\
TAG & $\geq 150$ & 36,3 \\
IMC & $\geq 27$ & 33,8 \\
\hline
\end{tabular}

* Valores tomados del Programa de Educación sobre colesterol de los Estados Unidos. **valor de referencia (17).
$(33,8 \%)$, mientras que muy pocos $(5,6 \%)$ presentaron aumento del C-LDL.

El cuadro 2 presenta la media y la desviación estándar de los valores del perfil lipídico y el índice arterial de la población en estudio, discriminados por intervalos de edad. Los datos más interesantes en esta tabla los dan los triglicéridos, el índice arterial (IA) y C-HDL. En toda la población, los TG y el IA están por encima de los niveles deseables ( $<150 \mathrm{mg} / \mathrm{dL}$ para los TG y $(<3,5$ para el IA); el C-HDL, en la mayoría está por debajo de 35 $\mathrm{mg} / \mathrm{dL}$, excepto para el grupo más joven, lo que muestra una población en términos generales en alto riesgo de sufrir EC, independientemente de las cifras de colesterol.

El cuadro 3 muestra la distribución por porcentajes de las alteraciones del perfíl lipídico en los intervalos de edad. Los datos revelan que es el intervalo de edad de 31 a 40 años donde se presenta el mayor porcentaje de alteraciones del perfil lipídico con $48 \%$ de TG altos y $79 \%$ de C-HDL bajos. También es interesante observar en el cuadro 3 el aumento de los niveles de colesterol con la edad. En el grupo de edad de 51-60 años, 55,5\%

Cuadro 3. Distribución porcentual de alteraciones en el perfil lipídico por intervalos de edad.

\begin{tabular}{|c|c|c|c|c|}
\hline \multirow[t]{2}{*}{$\begin{array}{l}\text { Intervalos } \\
\text { de edad }\end{array}$} & \multicolumn{4}{|c|}{$\begin{array}{l}\text { Porcentaje de individuos con } \\
\text { alteraciones del perfil lipídico }\end{array}$} \\
\hline & TAG & CT & C-HDL & C-LDL \\
\hline $20-40$ & 38,4 & 7,6 & 53,8 & 0 \\
\hline $31-40$ & 48,2 & 20,6 & 79,3 & 6,8 \\
\hline $41-50$ & 30,0 & 10,0 & 70,0 & 0 \\
\hline $51-60$ & 33,3 & 55,5 & 55.5 & 11.1 \\
\hline$>61$ & 10,2 & 20,4 & 50,1 & 10,1 \\
\hline
\end{tabular}

Cuadro 2. Media y desviación estándar del perfíl lipídico por intervalos de edad.

\begin{tabular}{lrcrrr}
\hline Perfil lipídico & \multicolumn{4}{c}{ Intervalo de edad (en años) } \\
& $20-30\left(13^{\star}\right)$ & $31-40(29)$ & $41-50(10)$ & $51-60(9)$ & $>61(10)$ \\
\hline CT & $167,3 \pm 22$ & $171,8 \pm 32$ & $75,6 \pm 26$ & $201,2 \pm 48$ & $173,8 \pm 38$ \\
C-HDL & $40,4 \pm 29$ & $33,0 \pm 12$ & $34,4 \pm 9,6$ & $31,6 \pm 5$ & $34,4 \pm 12$ \\
C-LDL & $88,5 \pm 32$ & $102,3 \pm 30$ & $104,0 \pm 18$ & $127,5 \pm 43$ & $111,2 \pm 34$ \\
TAG & $190,3 \pm 115$ & $207,8 \pm 120$ & $178,2 \pm 140$ & $205,4 \pm 152$ & $137,1 \pm 50$ \\
Ind. Arterial & $5,3 \pm 2,4$ & $5,5 \pm 1,7$ & $5,4 \pm 1,5$ & $6,6 \pm 2,4$ & $5,6 \pm 2,4$ \\
\hline
\end{tabular}


de los individuos tenían colesterol por encima de $200 \mathrm{mg} / \mathrm{dL}$.

El cuadro 4 indica la relación entre el perfil lipídico (variables dependientes) y algunos factores de riesgo no lipídicos (variables independientes) de sufrir enfermedad cardiovascular.

En la muestra del estudio, los factores de riesgo no lipídicos que más incidieron en los componentes del perfil lipídico fueron el índice de masa sobre los triglicéridos, con una probabilidad de 0,006 ; en el CT, el hábito de fumar con probabilidad de 0,02 ; en el C-HDL, inciden los antecedentes familiares con una probabilidad de 0,05 y el índice de masa con una probabilidad de 0,03. En el C-LDL, los factores más incidentes fueron la edad con una probabilidad de $0,07 \mathrm{y}$ el hábito de fumar con probabilidad de 0,08 .

\section{Discusión}

La enfermedad cardiovascular es la causa principal de muerte en los países occidentales. Además de los factores de riesgo lipídicos que predisponen a sufrir ECV, son bien conocidos algunos de los factores no lipídicos que predisponen a la ECV, incluyendo el tabaquismo, la obesidad, el consumo elevado de grasas de origen animal (en la carne o en los productos lácteos), la hipertensión arterial y los antecedentes familiares de ECV.

Diferentes estudios han clasificado la dieta como un importante factor de riesgo para ECV; se conoce, por ejemplo, que en Japón, China y otras partes de oriente, la dieta es relativamente baja en grasas saturadas y la prevalencia de ECV también es baja $(12,13)$.
Por otro lado, el trabajo de Grundy (14) indica que la alta ingesta en grasas poliinsaturadas reduce el nivel de colesterol plasmático. Esta reducción ocurre principalmente en lipoproteína de baja densidad; nuestros resultados correlacionan con esta información ya que se encontró que esta población no tiene un alto consumo en grasas saturadas; bajo estas condiciones, sólo 2,8\% está en alto riesgo debido a la dieta (figura 1) y la incidencia de la misma como riesgo de EC no fue significativa (cuadro 4). Si la dieta baja en grasas saturadas contribuye a la disminución de C-LDL, esto podría explicar el bajo porcentaje $(5,6 \%)$ de individuos del estudio que presentan C-LDL alta.

Se conoce que el nivel de colesterol total en los hombres aumenta con la edad y con ella el riesgo de EC. Por ejemplo, en un hombre de 62 años, el riesgo de morir por ECV es 500 veces mayor que en un hombre de 22 años, aunque los niveles de colesterol LDL sean iguales para ambos (11). Esta misma relación se encuentra en nuestro estudio donde el nivel más alto de colesterol total (201 \pm $47,7)$ se presenta en el intervalo de edad comprendido entre 51 y 60 años. Sin embargo, la población joven de este estudio (31-40) presenta otros factores de riesgo diferentes a los niveles circulantes de colesterol.

El índice arterial CT/C-HDL ha mostrado ser mejor predictor de ECV que CT solo, pues muestra la relación entre los niveles de CT con C-HDL aun cuando el CT esté normal; en nuestro estudio, el nivel de C-HDL está determinando el riesgo. El índice arterial resulta un valioso dato pronóstico para determinar si el C-HDL está ejerciendo pro-

Cuadro 4. Incidencia de los factores de riesgo no lipídicos sobre el perfíl lipídico.

\begin{tabular}{lcccccc}
\hline Variables & \multicolumn{7}{c}{ Factores de riesgo no lipídicos } & Anticas & Antecedentes & $\begin{array}{c}\text { Dieta } \\
\text { familiares }\end{array}$ & & $\begin{array}{c}\text { Presión } \\
\text { sistólica }\end{array}$ & $\begin{array}{c}\text { Indice de } \\
\text { masa }\end{array}$ & Edad & Fumar \\
\hline CT & 0,20 & 0,94 & 0,59 & 0,14 & 0,15 & $0,02^{\star}$ \\
C-HDL & $0,05^{\star}$ & 0,87 & 0,45 & $0,03^{\star}$ & 0,65 & 0,91 \\
C-LDL & 0,50 & 0,41 & 0,87 & $0,03^{\star}$ & 0,07 & $0,08^{\star}$ \\
TAG & 0,62 & 0,70 & 0,23 & $0,006^{\star}$ & 0,56 & 0,94 \\
\hline
\end{tabular}


tección adecuada; los resultados muestran que, para la población, resultó ser mayor de 5 lo cual la clasifica como una población de alto riesgo por C-HDL bajos, independiente de los niveles de colesterol en algunos casos normales.

Es importante destacar que esta población presentó un factor de riesgo también muy preocupante, las altas concentraciones plasmáticas de TG (cuadro 1). En muchos estudios prospectivos y de casos y controles, los niveles de TG plasmáticos correlacionan positivamente para EC (15).

Los niveles elevados de TG con frecuencia están asociados a niveles reducidos de C-HDL (6) y, por extensión, si los bajos niveles de C-HDL son aterogénicos, los elevados niveles de TAG asociado a los bajos C-HDL podrán ser considerados aterogénicos (16).

Estos trabajos sustentan los resultados encontrados por nosotros para la población objeto de este estudio en donde los factores de riesgo importantes fueron los altos niveles de TAG y los bajos de C-HDL que, a su vez, también confirman otros hallazgos en poblaciones colombianas que muestran la tendencia de nuestra población a padecer de hipertrigliceridemia aislada (9).

La incidencia de los factores de riesgo no lipídicos (fumar, índice de masa corporal, hipertensión, etc.) (cuadro 4) en esta población, no se mostraron de manera diferente a lo informado en la literatura; el fumar incidió sobre los niveles de colesterol total y C-LDL; los antecedentes familiares y el índice de masa corporal en el C-HDL, y el índice de masa corporal en los TG; esto indica, en términos generales, que la población de estudio no se aleja del comportamiento de la población mundial en cuanto a los factores de riesgo a sufrir EC, pero sí alarma y son determinantes los hallazgos sobre la hiper-trigliceridemia asociada a bajos niveles de C-HDL. Sería importante para obtener datos más concluyentes adelantar estudios de perfil lipídico con poblaciones más grandes y hacer un diseño experimental que arroje datos sobre posibles causas de las hipertrigliceridemia no asociadas al consumo de grasas saturadas, en la población colombiana.

\section{Agradecimientos}

Las autoras agradecen a la Facultad de Formación Avanzada e Investigaciones de la Universidad del Quindío por su apoyo financiero y su valiosa colaboración. Igualmente, agradecemos a todo el personal del Centro de Investigaciones Biomédicas Manuel Elkin Patarroyo y a la licenciada Miryan Beltrán del Laboratorio de Bioquímica.

\section{Referencias}

1. Castelli WP, Garrison RJ, Wilson PWF, Abott RD, Kalousdian S, Kannel WB. Incidence of coronary heart disease and lipoprotein cholesterol levels; the Framminghan Study. JAMA 1986;256:2835-8.

2. Anderson KM, Castelli WP, Levy D. Cholesterol and mortality 30 years of follow-up of the Framminghan study. JAMA 1987;257:2176-80.

3. Stamler J, Wenworth D, Neaton JD. Is the relationship between serum cholesterol and risk of premature death from coronary heart disease continuous grade? Findings in 356,220 primary screens of multiple risk Factor Intervention Trial MRFIT. JAMA 1986;256:2823-8.

4. Halley SR, Rossemman RH, Bawol RD, Brand RJ. Epidemiology as guide to clinical decisions. The association between triglyceride and coronary heart disease. N Eng J Med 1980;302:1383-9.

5. Castelli WP Editorial. Cardiovascular Risk Factor 1994.

6. Davidoff P. Cholesterol and triglycerides in atherosclerosis: epidemiologic and physiopathologic considerations. Rev Med Chile 1991;119:1050-8.

7. Sniderman A, Vu H, Cianflone K. Effect of moderate hypertrigliceridemia on the relation of plasma total and LDL apo B levels. Atherosclerosis 1991;89:109-16.

8. ILIB. Latinoamérica Recomendaciones de ILIB para el diagnostico de las dislipidemias en Latinoamérica (Panel de expertos en detección, evaluación y tratamiento de dislipidemias del International Lipid Information Bureau (ILIB). Latinoamerica Cardiovascular Risk Factor 1994;10-27

9. Consenso Colombiano de Dislipoproteinemias. Sociedad Latinoamericana de Aterosclerosis. Capítulo colombiano. Boletín informativo 1992;2-3:6-7

10. Cediel V, Rodríguez J. Glicemia y perfil lipídico en un grupo de personas aparentemente sanas de la ciudad de Pereira. Rev Med Risaralda 1995;1:3-6.

11. National Cholesterol Education Program. Second Report of the National Cholesterol Education Program. 
Expert Panel on detection, evaluation, and treatment of High Blood Cholesterol in adults. Circulation 1994; 89:1331-45.

12. Kagan A, Harris BR, Winkelstein W Jr, Johnson Kg, Kato H, Syme SL, Rhoads GG, Gay MI, Nichman MZ, Hamilton HB, Tillotson J. Epidemiologic studies of coronary heart disease and stroke in Japan, Hawail and California: demographic, physical, dietary and biochemical characteristics. J Chron Dis 1974;27:345-64.

13. People's Republic of China-United States Cardiovascular and Cardiopulmonary Epidemiology Research Group. An epidemiology study of cardiovascular and cardiopulmonary disease risk factors in four populations in the People's Republic of China: baseline report from PRCUSA [Collaborative Study]. Circulation 1992;85:1083-96.

14. Grundy SM. Terapia dietética para diferentes formas de hiperproteinemia. Circulation 1987;76:523-8.

15. Austin. Plasma triglycerides and coronary heart disease. Arterioscler Throm 1991;11:2-14.

16. Grundy SM, Vega GL. Two different views of the relationship of hypertriglyceridemia to coronary heart disease. Arch Intern Med 1992;152:28-34.

17. Blanco de la Mora E. Peso corporal y su relación con mortalidad. Editorial Mundo Médico 1996. 